\title{
National mapping of soil-transmitted helminth and schistosome infections in Ethiopia
}

\author{
Gemechu Tadesse Leta ${ }^{1,2^{*}} \mathbb{D}$, Kalkidan Mekete ${ }^{1}$, Yonas Wuletaw ${ }^{1}$, Abeba Gebretsadik' ${ }^{1}$ Heven Sime ${ }^{1}$, \\ Sindew Mekasha ${ }^{1}$, Adugna Woyessa', Oumer Shafi ${ }^{3}$, Jozef Vercruysse², Jack E. T. Grimes ${ }^{4}$, lain Gardiner ${ }^{5}$, \\ Michael French ${ }^{6,7}$, Bruno Levecke ${ }^{2}$, Lesley Drake ${ }^{5}$, Wendy Harrison ${ }^{6}$ and Alan Fenwick ${ }^{6}$
}

\begin{abstract}
Background: An accurate understanding of the geographical distributions of both soil-transmitted helminths (STHs; Ascaris lumbricoides, Trichuris trichiura, and the hookworms Necator americanus and Ancylostoma duodenale) and schistosomes ( $\mathrm{SCH}$; Schistosoma mansoni and S. haematobium) is pivotal to be able to effectively design and implement mass drug administration (MDA) programmes. The objective of this study was to provide up-to-date data on the distribution of both STH and SCH in Ethiopia to inform the design of the national control program and to be able to efficiently achieve the $75 \%$ MDA coverage target set by the WHO.
\end{abstract}

Methods: Between 2013 and 2015, we assessed the distributions of STH and SCH infections in a nationwide survey covering 153,238 school-aged children (aged 5-15 years), from 625 woredas (districts), representing all nine Regional States and two City Administrations of Ethiopia. Nationwide disease maps were developed at the woreda level to enable recommendations on the design of the national MDA programme.

Results: The prevalence of any STH infection across the study population was $21.7 \%$, with A. lumbricoides (12.8\%) being the most prevalent STH, followed by hookworms (7.6\%) and T. trichiura (5.9\%). The prevalence for any SCH was $4.0 \%$ in areas where both SCH species were evaluated. Schistosoma mansoni was the most prevalent SCH (3.5 vs 0.3\%). STHs were more prevalent in southwest Ethiopia, whereas SCH was found mostly in the west and northeast of the country. The prevalence of moderate-to-heavy intensity infections was 2.0\% for STHs and 1.6\% for SCH. For STH, a total of 251 woredas were classified as moderately $(n=178)$ or highly endemic $(n=73)$, and therefore qualify for an annual and biannual MDA program, respectively. For $\mathrm{SCH}, 67$ woredas were classified as endemic and 8 as highly endemic, and hence they require every two years and annual MDA programme, respectively.

Conclusions: The results confirm that Ethiopia is endemic for both STHs and SCH, posing a significant public health problem. Following the WHO recommendations on mass drug administration, 18 and 14 million school-aged children are in need of MDA for STHs and SCH, respectively, based on the number of SACs that live on the eligible geographical areas.

Keywords: Soil-transmitted helminthiasis, Schistosomiasis, Ascaris, Trichuris, hookworms, Schistosoma mansoni, Schistosoma haematobium, Woreda (districts), Disease maps, School-aged children, Endemicity, Mass drug administration

\footnotetext{
*Correspondence: gemechut_2006@yahoo.com

2 Department of Virology, Parasitology and Immunology, Faculty of Veterinary Medicine, Ghent University, Merelbeke, Belgium

Full list of author information is available at the end of the article
}

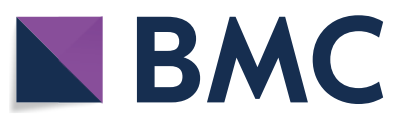

(c) The Author(s) 2020. This article is licensed under a Creative Commons Attribution 4.0 International License, which permits use, sharing, adaptation, distribution and reproduction in any medium or format, as long as you give appropriate credit to the original author(s) and the source, provide a link to the Creative Commons licence, and indicate if changes were made. The images or other third party material in this article are included in the article's Creative Commons licence, unless indicated otherwise in a credit line to the material. If material is not included in the article's Creative Commons licence and your intended use is not permitted by statutory regulation or exceeds the permitted use, you will need to obtain permission directly from the copyright holder. To view a copy of this licence, visit http://creativeco mmons.org/licenses/by/4.0/. The Creative Commons Public Domain Dedication waiver (http://creativecommons.org/publicdomain/ zero/1.0/) applies to the data made available in this article, unless otherwise stated in a credit line to the data. 


\section{Background}

Neglected tropical diseases (NTDs) are a diverse group of bacterial, parasitic and viral communicable diseases. They are widespread in tropical and subtropical countries where poverty, inadequate sanitation and hygiene are common [1]. A group of these NTDs can be controlled by either innovation or intensified disease management at the level of the individual patient, or through mass drug administration (MDA) to the most at-risk populations. Some of the MDA-amenable parasites include the soil-transmitted helminths (STHs): Ascaris lumbricoides, Trichuris trichiura and the hookworms (Nectator americanus and Ancylostoma duodenale) and schistosomes (SCH: Schistsoma mansoni and S. haematobium). The MDA platforms for these NTDs are often based on deworming children at school. The recommended dosages for deworming are a single oral dose of albendazole $(400 \mathrm{mg})$ or mebendazole $(500 \mathrm{mg})$ for STHs, and praziquantel for $\mathrm{SCH}(40 \mathrm{mg} / \mathrm{kg}$; the weight often approximated by height using a dose-pole [2]). The global community has committed to cover at least $75 \%$ of the school-aged children (SAC) in endemic areas for both STHs and SCH by 2020 [3], with the ultimate goal to reduce the prevalence of heavy intensity $(\mathrm{HI}$, in case of $\mathrm{SCH}$ ) or moderate-to-heavy intensity (MHI, for STH) infections, as defined by the WHO [4]. These are thought to be the intensity of infections that cause the highest morbidity. The frequency and populations targeted for MDA are determined by the prevalence in each implementation unit. For STHs, it is recommended to distribute drugs twice a year when the prevalence of any STHs exceeds $50 \%$, and once a year when the prevalence is at least $20 \%$. For prevalence below $20 \%$, a case-by-case treatment is recommended [4]. For $\mathrm{SCH}$, an annual round of MDA is recommended when the prevalence is $\geq 50 \%$, every two years when the prevalence is $\geq 10 \%$. For any other prevalence of $\geq 1 \%$, twice MDA during the primary school years is recommended [2].

Both STHs and SCH infections pose an important threat to public health in Ethiopia [5-9]. In 2012, it was concluded that Ethiopia represents one of the top five sub-Saharan countries with the highest prevalence of STHs (second place for Ascaris, third place for hookworm and fourth for Trichuris). For SCH, Ethiopia has the 14th highest prevalence among sub-Saharan countries [10]. Across the country, there have been a series of disease distribution studies, reporting a wide range of infection $[5,6,9,11,12]$. However, these studies are not ideal for making recommendations on the best MDA strategy at a national level. They are generally dated (early 1970 s to 2015 ; $[5,9,13])$, nor do they cover the entire country.
Moreover, they differ considerably in sample size, design and diagnostic method utilized hindering direct comparison $[9,12,14,15]$, which further complicates effectively designing and implementing MDA programs. Since 2000, the Ethiopian government has implemented STH deworming of children in under five as part of an integrated vitamin A supplementation programme. For $\mathrm{SCH}$, the country has historically focused on a case-based treatment of laboratory-confirmed patients. Following the London Declaration on NTDs in 2012 [16], the Federal Ministry of Health of Ethiopia (FMoH) developed an NTD Master Plan and Roadmap for combating the country's most common NTDs [17]. For STHs and SCH, the target is to control morbidity through the reduction of MHI infections by means of MDA. Up-to-date data on disease distribution are therefore pivotal to ensure the area's most in need of MDA are covered and to avoid the initiation of large-scale deworming in areas where disease is rare or absent $[4,18]$.

Here, we describe the distribution of STHs and SCH in 153,238 SAC, across 2989 schools in 625 woredas, across all nine Regions and two City Administrations in Ethiopia. We present disease distribution maps to inform the design of the national programme.

\section{Methods \\ Study area}

In 2015, the Ethiopia's population was estimated at 100 million [19]. The country has three administrative levels. The first level includes nine Regional States: Afar, Amhara, Benishangul-Gumuz, Gambela, Harari, Oromia, Southern Nations, Nationalities and Peoples' Region (SNNPR), Somali and Tigray; and two chartered City Administrations: Addis Ababa and Dire Dawa. The second and the third administrative levels are zones and woredas (districts), respectively. Woredas are the implementation units for NTD control programmes in Ethiopia.

The distribution of STHs and SCH was mapped over two surveys. In the first survey (November 2013 to March 2014), eight of the nine Regional States and one of the two City Administrations were included. In the second survey (February and April 2015), Amhara and Addis Ababa were mapped. In addition, the second survey included fine-scale mapping survey in Somali Regional State with the aim to cover woredas that were not mapped in the first survey.

\section{Field procedures}

For each woreda (or sub-city in the case of City Administrations), ten schools were randomly selected from the 
list of elementary schools provided by the Federal Ministry of Education (FMoE). From these, five schools were purposively selected by the Woreda Health Office, biased towards schools thought to be at-risk for $\mathrm{SCH}$ infections (due to proximity to water bodies, reports on $\mathrm{SCH}$ infections, irrigation and fishing practices of the community). An exception to this rule were those woredas where the safety of the field teams during the survey could not be secured. This was of particular impact in Somali Region and was compounded by the relatively small numbers of children enrolled per school. This is evidenced in the number of people surveyed. For the later determination of treatment approach, in these areas the nearest adjacent woreda was used to decide MDA.

Once the school selection was completed, the field team made the necessary pre-visit arrangements with the school directors. On the day of visit, all students of grade 5 (children around 12 years of age) were arranged in two lines, one for girls and one for boys. A random selection was made of 25 girls and 25 boys, resulting in a total maximum of 50 students per school. In schools with fewer than 25 boys or girls in the appropriate grade, children from lower grades (grade four: children around 11 years of age) or higher grade (grade six: children around 13 years of age) were included. The selected students were asked to provide both a stool and a urine sample.

Stool samples were screened for the presence of STHs and $S$. mansoni eggs, applying a single Kato-Katz thick smear [20]. The number of eggs of STHs (A. lumbricoides, T. trichiura and hookworms) and S. mansoni were multiplied by 24 to obtain the faecal egg counts (FECs) expressed in eggs per gram of stool (EPG) for each of the four helminth species.

Urine specimens were screened for S. haematobium in two consecutive steps. First, the presence of haematuria was assessed using Haemastix ${ }^{\circledR}$ (Bayer HealthCare LLC, Elkhart, Indiana, USA). The results of this test were recorded as either negative, trace,,+++ or +++ . Subsequently, urine samples in which at least a trace of haematuria was detected, were subjected to urine filtration to assess the number of $S$. haematobium eggs in $10 \mathrm{ml}$ of urine.

To ensure the quality of the parasitological results, the field team were instructed to read slides within $30 \mathrm{~min}$ to avoid over-clearing of hookworm eggs. For each team, we assigned a team leader to read $10 \%$ of the Kato-Katz slides and give feedback on a timely basis to the field team. Given challenges of hookworm egg degradation with time, hookworm eggs were not used for quality control purposes.
In addition, a questionnaire was conducted to collect information on water, sanitation and hygiene (WASH) at the school level. The results of this questionnaire and parasite infection for the first round of survey were reported elsewhere by Grimes et al. [21].

\section{Training of the field teams}

In total, 54 field teams from the different Regional States and City Administrations were involved in this survey. Each team consisted of one health officer (for the questionnaires and treatment) and three laboratory technicians (for the examination of stool and urine samples). The training was provided at the Ethiopian Management Institute (EMI) in Bishoftu, Oromia regional state. During these trainings, both theoretical and practical sessions were given to 164 health officers and laboratory technicians. The theoretical sessions focussed on a variety of aspects of the diseases (life-cycles, pathogenesis, laboratory diagnosis, prevention and control strategies), while the practical sessions focussed on the diagnostic methods (the use of Haemastix ${ }^{\circledR}$, urine filtration and Kato-Katz thick smear), archiving Kato-Katz slides for quality control, completing questionnaires on WASH and using LINKS $^{\circledR}$ (a smart phone-based application to collect data).

\section{Mapping coordination and supervision}

Additional file 1: Figure S1 provides an overview of the coordination and supervision of this study. The Ethiopian Public Health Institute (EPHI), the technical arm of $\mathrm{FMoH}$, was responsible for the overall coordination of the survey. For this coordination, four central supervisors were assigned. At the Regional States and City Administrations, at least one supervisor from either the Regional Health or Education Office for each Regional State (and City Administration) was assigned throughout the mapping period. In addition, external supervisors from the Ugandan Vector Control Division (Uganda) and the Kenyan Medical Research Institute (Kenya) conducted monitoring visits. These supervisors independently evaluated the survey coordination and communication flow between the central level (EPHI), the regional supervisors (Regional States and City Administration) and the field teams. They also monitored the operational procedures at the schools, providing feedback to the field teams, correcting those that were not adhering to the mapping protocol.

\section{Data collection and data management}

Data were collected using the LINKS $^{\circledR}$ data collection system developed by the Task Force for Global Health 
(Atlanta, USA). This is an android-based application that allows standardized entry of epidemiological data across the different teams. At the school level, the teams collected the GPS coordinates, the total number of students, the total number of boys and girls in the school, the availability of toilets, water supplies and hand washing facilities. At the individual level, the teams collected the age, sex, the number of Ascaris, Trichuris, hookworm, S. mansoni eggs, the presence of haematuria, and the number of S. haematobium eggs. Raw data were downloaded from the LINKS system server and were subsequently curated using Microsoft Excel (Microsoft Corporation, Redmond, WA, USA).

\section{Statistical data analysis}

The prevalence and intensity of infections were calculated for any STH and $\mathrm{SCH}$, and the individual helminth species, separately. Prevalence was estimated by the proportion of children for whom eggs of a particular helminth species were detected. Intensity of infection was measured as the prevalence of $\mathrm{MHI}$ infections were calculated for any $\mathrm{STH}$ and $\mathrm{SCH}$, and for the different helminth species separately [4]. Unweighted prevalence was calculated at both regional and woreda level. To this end, the proportion of children excreting eggs over the total number screened in the region or woreda was calculated. Subsequently, the point estimates of the different parameters were plotted on a geographical map of Ethiopia using ArcGIS version 10.4 (ESRI, Inc., Redlands, USA).

These infection parameters (prevalence, infection intensity and mixed infections) were calculated at the different administrative levels (national, Regional States/ City Administrations, woredas and schools). Subsequently, the point estimates of the different parameters were plotted on a geographical map of Ethiopia using ArcGIS version 10.4 (ESRI, Inc.). We explored the variation in infections of any intensity and MHI by generalized linear mixed models, which were fitted for each of the five helminth species with the presence/absence of infections of any intensity or MHI infections as outcome, the age (in years), sex (2 levels: boy and girl) and Region/City Administration (11 levels: Afar, Amhara, BenishangulGumuz, Gambela, Harari, Oromiya, Southern Nations, Nationalities and Peoples' Region (SNNPR), Somali, Tigray (Regions), Addis Ababa and Dire Dawa (City Administrations)) as explanatory variables. In the analysis, we accounted for clustering of children from the same school and schools from the same woreda. To facilitate an easy interpretation, we centralized the age around its median (12 years) and converted into a binary outcome (boy $=0$, girl $=1$ ). The level of significance was set at $P$ $<0.05$.
In addition, we determined the prevalence of mixed infections (the proportion of children who were excreting eggs of at least two different helminths). Finally, we determined the MDA design for each of the woredas included in this survey. To this end, we classified the woredas into low, moderate and high endemic for STHs and SCHs applying the WHO classification criteria [4] on the prevalence of any STH and SCH infection across the five schools of the same woreda (the proportion of cases over all children screened in that woreda).

Quality control result analysis for the Kato-Katz thick smear Quality control of the FECs of any STHs and S. mansoni was performed for 6042 individuals. For this, Kato-Katz thick smears were re-examined by a team leader. The proportions of both false positives and false negatives were assessed. To this end, we assumed that the team leader was correct. In cases where both results indicated presence of eggs, the agreement in egg counts was assessed. Result disagreements were defined when the difference in egg counts was greater than 10 when the team leader counted fewer than 100, or when the difference in egg counts was more than $20 \%$ when the team leader counted more than 100 eggs [22].

\section{Map creation}

The results from the field surveys were used to develop graphical maps of the distribution of $\mathrm{SCH}$ and $\mathrm{STH}$ infection, and of $\mathrm{SCH}$ and $\mathrm{STH}$ moderate and high intensity infections. This was done in ArcGIS version 10.4 (ESRI, Inc.). The maps are intended as a useful geographical guide of the distribution of infection. There were challenges with representing the woreda-level results from the field surveys onto a graphical map, meaning a one-to-one representation was not possible. This was related particularly to the rapid increase in the number of woredas in the country (resulting in splits between 'mother' and 'daughter' districts and subsequent changes to administrative boundaries) and the lag in availability of updated mapping software to represent these accurately. This means that the graphical maps cannot be a perfect one-to-one representation of the data collected. This is an inherent challenge in countries with changing administrative borders and sub-divisions. The numbers of woredas in the tables for each infection category are most accurate and used to inform the control program.

\section{Informing MDA strategy}

In order to determine the MDA strategy in each district, the upper $95 \%$ confidence interval of woreda (district) level prevalence was taken of either 'any STH' or 'any SCH.' This was then compared to the WHO-recommended cut-offs [2]. Using the upper confidence interval 
rather than the point prevalence represents a conservative approach to ensure the program reaches as many people in need of treatment as possible.

\section{Results}

\section{Study population}

Stool samples were collected from 153,238 SAC from 2989 schools in 625 woredas across the nine Regional States and two City Administrations for both STH and $S$. mansoni infections. Urine samples were also collected from 112,218 SAC attending 2226 schools in 466 woredas across the 9 Regional States and 2 City Administrations. In both datasets, the age of the children ranged from five to 15 years, with a median age of 12 years. The sexes were generally equally represented (ratio of males/females $=$ 0.99).

In the following paragraphs we summarize the prevalence of infections of any intensity and MHI for STHs and $\mathrm{SCH}$, separately. Then we discuss the occurrence of mixed STH and $\mathrm{SCH}$ infections. Finally, we provide an overview of the recommended MDA strategy at the level of the woreda for both STHs and SCH.

\section{Prevalence of STH infections of any intensity and MHI}

The unweighted prevalence of STH infections of any intensity and MHI intensity across the Regions/City Administrations, sex and age are summarized in Table 1. The overall unweighted prevalence of infection with any STH was $21.7 \%$. The most prevalent STH species was A. lumbricoides (12.8\%), followed by hookworms (7.6\%) and T. trichiura (5.9\%). There was a large variation in prevalence across the different Regional States and City Administrations. For any STH infection, the prevalence ranged from 1.7\% (Addis Ababa City) to 58.1\% (Gambela). For the different STH species separately the prevalence ranged from $0.1 \%$ (Harari) to $45.2 \%$ (Gambela) for Ascaris, from 0.0\% (Addis Ababa City Administration) to $21.5 \%$ (Gambela) for hookworms, and from $0.1 \%$ (Afar, Dire Dawa City Administration and Harari) to 15.3\% (SNNPR) for T. trichiura. Figure 1 illustrates the prevalence at the woreda level for any STH infection. In general, the distribution of any STH infection is more prevalent in the south (SNNPR), southwest (Gambela, Oromiya and Benishangul-Gumuz) and north (Amhara and Tigray) of Ethiopia and less prevalent in the east of the country (Afar).

The variation in any STH infections across sex was small. For example, the prevalence of any STH infections was equally distributed across both sexes $(21.6 \%$ for sex $=0 v s 21.8 \%$ for sex $=1$ ), and this absence of any variation across sex was also observed for the different STH species, separately (Ascaris: 12.8 vs 12.8\%; Trichuris: 5.9 vs 5.9\%; hookworms: 7.6 vs $7.6 \%)$. The variation in prevalence was more clear across the different age categories. The prevalence of any STH infection ranged from $21.1 \%$ in SAC of 11 years-old to $30.3 \%$ in SAC of 15 years-old. Across the different STH species, this distinct variation was only observed for Ascaris infections (SAC of 11 years-old: $11.8 \%$ vs SAC of 15 years-old: $22.7 \%)$. For the other STH species, the variation in prevalence was less pronounced, the difference in prevalence across the age categories not exceeding 3.5 percentage points (Trichuris: 4.7 vs $7.9 \%$; hookworms: 7.3 vs $8.5 \%$ ).

The prevalence of MHI intensity of any STH infection was $2 \%$. For the STH species separately, the proportions were $1.7 \%, 0.2 \%$ and $0.1 \%$ for Ascaris, Trichuris and hookworms, respectively. SNNPR showed the highest prevalence of MHI infections for each of the three STH helminths (Ascaris: 5.2\%; Trichuris: $0.3 \%$ and hookworm: $0.2 \%)$. In seven out of 11 Regions/City Administrations no MHI infections were observed. Figure 2 illustrates the distribution of MHI infections for A. lumbricoides (Fig. 2a), T. trichiura (Fig. 2b), and hookworms (Fig. 2c) over the different woredas. The southern (SNNPR), south-western (Gambela, Oromiya and BenishangulGumuz) and northern (Amhara and Tigray) parts of the country have more infections of MHI. The variation in MHI infections was absent across both sexes and small across the different age categories. The results of the generalized linear mixed models confirmed the significant differences across Regions/City Administrations in the prevalence of STH infections of any intensity for each of the STH species. No significant differences in prevalence were observed for either sex or age. For the prevalence of $\mathrm{MHI}$ infections, the model did not convert.

\section{Prevalence of $\mathrm{SCH}$ infections of any intensity and $\mathrm{MHI}$ intensity}

The unweighted prevalence of $\mathrm{SCH}$ infections of any intensity and MHI intensity across the Regions/City Administrations, sex and age are summarized in Table 2. Across the subjects for whom both stool and urine were screened, the prevalence of infections of any $\mathrm{SCH}$ equalled $4.1 \%$. Based on the $\mathrm{SCH}$-specific data sets, $S$. mansoni was the most prevalent of both $\mathrm{SCH}$ species (3.5 vs $0.3 \%)$. As for STH infections, there was a large variation in prevalence across the different Regional States and City Administrations. For S. mansoni, the prevalence of any infection ranged from $0.1 \%$ (Addis Ababa City Administration) to $14.9 \%$ (Benishangul-Gumuz). Schistosoma haematobium was absent in 6 of the 11 Regions/ City Administrations (Harari, Oromiya, SNNPR, Tigray, Addis Ababa and Dire Dawa) and highly prevalent in Somali (18.5\%). The distribution of S. mansoni and S. haematobium infections across the different woredas is illustrated in Fig. 3. Overall, the variation in $\mathrm{SCH}$ infections 
Table 1 Variation in prevalence of soil-transmitted helminth infections across regions/city administrations, sex and age

\begin{tabular}{|c|c|c|c|c|c|c|c|c|c|}
\hline & \multirow[t]{2}{*}{$n$} & \multicolumn{2}{|l|}{ Any STH (\%) } & \multicolumn{2}{|c|}{ A. lumbricoides (\%) } & \multicolumn{2}{|l|}{ T. trichiura (\%) } & \multicolumn{2}{|l|}{ Hookworm (\%) } \\
\hline & & Any intensity & $\mathrm{MHI}$ & Any intensity & $\mathrm{MHI}$ & Any infection & $\mathrm{MHI}$ & Any infection & $\mathrm{MHI}$ \\
\hline \multicolumn{10}{|c|}{ Region/City Administration } \\
\hline Addis Ababa & 2718 & 1.7 & 0.0 & 1.2 & 0.0 & 0.6 & 0.0 & 0.0 & 0.0 \\
\hline Afar & 2377 & 2.2 & 0.0 & 2.0 & 0.0 & 0.1 & 0.0 & 0.2 & 0.0 \\
\hline Amhara & 25,246 & 14.3 & 0.5 & $8.0^{* * *}$ & 0.4 & 0.6 & 0.0 & $6.5^{* * *}$ & 0.0 \\
\hline Benishangul-Gumuz & 3109 & 10.3 & 0.0 & 0.8 & 0.0 & 0.7 & 0.0 & $9.3^{* * *}$ & 0.0 \\
\hline Dire Dawa & 1701 & 2.3 & 0.0 & 1.6 & 0.0 & 0.1 & 0.0 & 0.8 & 0.0 \\
\hline Gambela & 2977 & 58.1 & 0.6 & $45.2^{* * *}$ & 0.6 & 6.3 & 0.0 & $21.5^{* * *}$ & 0.1 \\
\hline Harari & 1804 & 3.0 & 0.0 & $0.1^{* *}$ & 0.0 & 0.1 & 0.0 & $2.8^{*}$ & 0.0 \\
\hline Oromia & 62,520 & 16.7 & 1.2 & $9.3^{* * *}$ & 0.9 & $4.6^{* *}$ & 0.2 & $5.6^{* * *}$ & 0.2 \\
\hline SNNPR & 37,544 & 42.4 & 5.7 & $25.9^{* * *}$ & 5.2 & $15.3^{* * *}$ & 0.3 & $13.5^{* * *}$ & 0.2 \\
\hline Somali & 966 & 38.0 & 0.0 & $36.4^{* * *}$ & 0.0 & 1.2 & 0.0 & $5.5^{* * *}$ & 0.0 \\
\hline Tigray & 12,276 & 5.4 & 0.1 & 2.2 & 0.0 & 0.2 & 0.0 & $3.3^{* * *}$ & 0.0 \\
\hline \multicolumn{10}{|l|}{ Sex } \\
\hline Sex 0 (male) & 76,385 & 21.6 & 1.9 & 12.8 & 1.7 & 5.9 & 0.1 & 7.6 & 0.1 \\
\hline Sex 1 (female) & 76,853 & 21.8 & 2.0 & 12.8 & 1.8 & 5.9 & 0.2 & 7.6 & 0.1 \\
\hline \multicolumn{10}{|l|}{ Age (in years) } \\
\hline $5-9$ & 1201 & 21.8 & 0.3 & 12.8 & 0.2 & 4.7 & 0.1 & 8.5 & 0.1 \\
\hline 10 & 23,962 & 21.2 & 1.7 & 11.8 & 1.4 & 5.8 & 0.1 & 8.0 & 0.2 \\
\hline 11 & 38,332 & 21.1 & 1.8 & 12.1 & 1.6 & 5.9 & 0.2 & 7.4 & 0.1 \\
\hline 12 & 45,447 & 21.8 & 2.3 & 13.0 & 2.0 & 6.2 & 0.1 & 7.3 & 0.1 \\
\hline 13 & 27,693 & 22.3 & 2.2 & 13.5 & 1.9 & 5.9 & 0.2 & 7.7 & 0.1 \\
\hline 14 & 15,342 & 22.0 & 1.6 & 13.4 & 1.4 & 5.2 & 0.1 & 8.1 & 0.1 \\
\hline 15 & 1261 & 30.3 & 2.6 & 22.7 & 2.4 & 7.9 & 0.2 & 8.3 & 0.1 \\
\hline Total & 153,238 & 21.7 & 2.0 & 12.8 & 1.7 & 5.9 & 0.2 & 7.6 & 0.1 \\
\hline
\end{tabular}

Notes: MHI: moderate-to-heavy intensity, comparisons to the baseline value (Region/City Administration: Afar; sex: sex 0; age $=12$ years) that reveal an odds ratio significantly different from one are indicated by at least one asterisk

${ }^{*} P<0.05,{ }^{* *} P<0.01,{ }^{* * *} P<0.001$

across both sex and age was small. The prevalence of infections of any intensity was equally distributed over sex for both SCH species (S. mansoni: 3.4 vs $3.6 \%$; S. haematobium: 0.3 vs $0.3 \%)$. Across the age categories, there was some increase over age infections, which was more apparent for S. haematobium. For this Schistosoma species, the prevalence ranged from $3.0 \%$ in SAC less than 9 years to $7.7 \%$ for children of 15 years of age. For S. haematobium, the variation in prevalence ranged from $2.9 \%$ for SAC of 11 years-old to $5.2 \%$ for SAC of 15 years-old.

Across the subjects for whom both stool and urine were screened, the prevalence of $\mathrm{MHI}$ infections of any $\mathrm{SCH}$ equalled 1.6\%. Based on the $\mathrm{SCH}$-specific data sets, the prevalence of MHI infections was $1.4 \%$ for $S$. mansoni and $0.1 \%$ for $S$. haematobium. The distribution of $S$. mansoni MHI infections across the Regions/City Administration followed a similar pattern as that observed for infections of any intensity, being high in Regions/ City Administrations where infections of any intensity are prevalent (ranging from $0.1 \%$ in Addis Ababa City
Administration and Afar to 5.2\% in Benishangul-Gumuz). For S. haematobium, MHI infections were highly prevalent in 3 Regional States (Somali, Gambella and Amhara), of which Somali reported the highest prevalence (11.9\%). The prevalence of S. mansoni and S. haematobium MHI infections across the different woredas is illustrated in Fig. 4. Schistosoma mansoni MHI infections were more prevalent in the west (Benishangul-Gumuz and Gambela) and the north of (Tigray), whereas MHI infections S. haematobium were more prevalent in the east of the country (Somali). The variation in MHI infections across sex was small for both SCH species. For age, the prevalence increased when children were older. The results of the generalized linear mixed models confirmed the significant differences across Regions/City Administrations in the prevalence of $S$. mansoni infections. No significant differences in prevalence were observed for either sex or age. For the prevalence of S. haematobium infections and MHI infections, the model did not convert. 


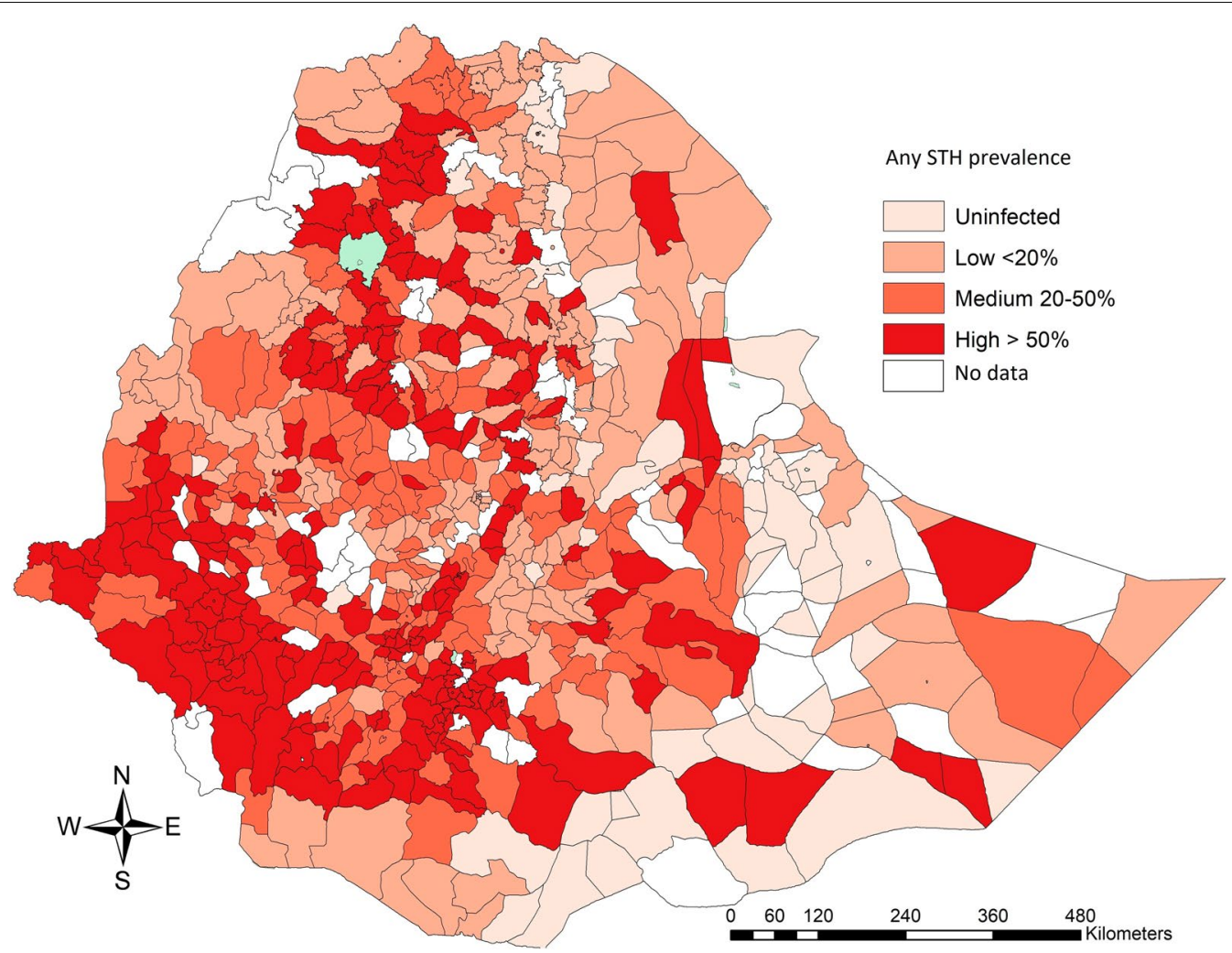

Fig. 1 The woreda prevalence of any soil-transmitted helminth infections in school-aged children, Ethiopia 2013-2015. The map illustrates the woreda-level prevalence of any soil-transmitted helminth infections in 153,238 school-aged children, Ethiopia 2013-2015. The woreda-level prevalence is based on the number of children, which were found to be positive based on single Kato-Katz thick smear, over the total number of children screened in a woreda. The source of the administrative boundaries is www.gadm.org/

\section{Mixed STH and SCH infections}

Of the 112,218 SAC for whom both a stool and a urine sample was processed, 5776 children harboured infections with two or more helminth species. Mixed infections with two, three, four and five different helminth species were observed in $87.4 \%, 11.8 \%, 0.8 \%$ and $0.02 \%$ of these children, respectively. The different mixed STH and $\mathrm{SCH}$ helminth infections are summarized in Table 3. The most prevalent mixed infections were Ascaris infections mixed with either Trichuris or hookworms, accounting for more than $50 \%$ of the mixed infections.

\section{Recommended MDA strategy for STH and SCH infections}

As outlined in the methods section, the woreda-level classification is based on the upper confidence interval of the woreda level estimate of 'any STH' or 'any SCH. Table 4 summarizes the endemicity of $\mathrm{STH}$ and $\mathrm{SCH}$ infections across the different woredas and the corresponding MDA strategy based on WHO guidelines [4]. For STHs, 178 out of $625(28.5 \%)$ woredas require MDA once a year and 73 $(11.7 \%)$ require MDA twice a year. For SCH, MDA once a year is recommended for $8(1.3 \%)$ woredas, once every two years for $67(10.7 \%)$ and once every three years for 190 woredas $(30.4 \%)$. Large-scale treatment for STH and SCH was not warranted for 37 and 360 woredas, respectively.

\section{Quality control of Kato-Katz thick smears}

In total, 6042 Kato-Katz thick smear slides were re-examined at random by the team leaders. Overall, there was an agreement in test result in $91 \%$ of the positive cases $(9 \%$ false negatives) and $98 \%$ of the negative cases $(<2 \%$ false positive cases). The numbers of false negatives were relatively high for Trichuris (8.7\%) and low for hookworms (3.0\%). Among the negative cases, there was an agreement in $93 \%$ of the cases. The agreement was high for $S$. mansoni (98.4\%) and relatively low for Ascaris (93.7\%).

\section{Discussion}

The WHO has set the ambitious goal to cover at least $75 \%$ of SAC in need of treatment against STHs and $\mathrm{SCH}$ infections in all endemic countries by 2020 [4, 23-25]. In order to roll out an MDA programme, detailed disease maps are pivotal to ensure that areas most in need 


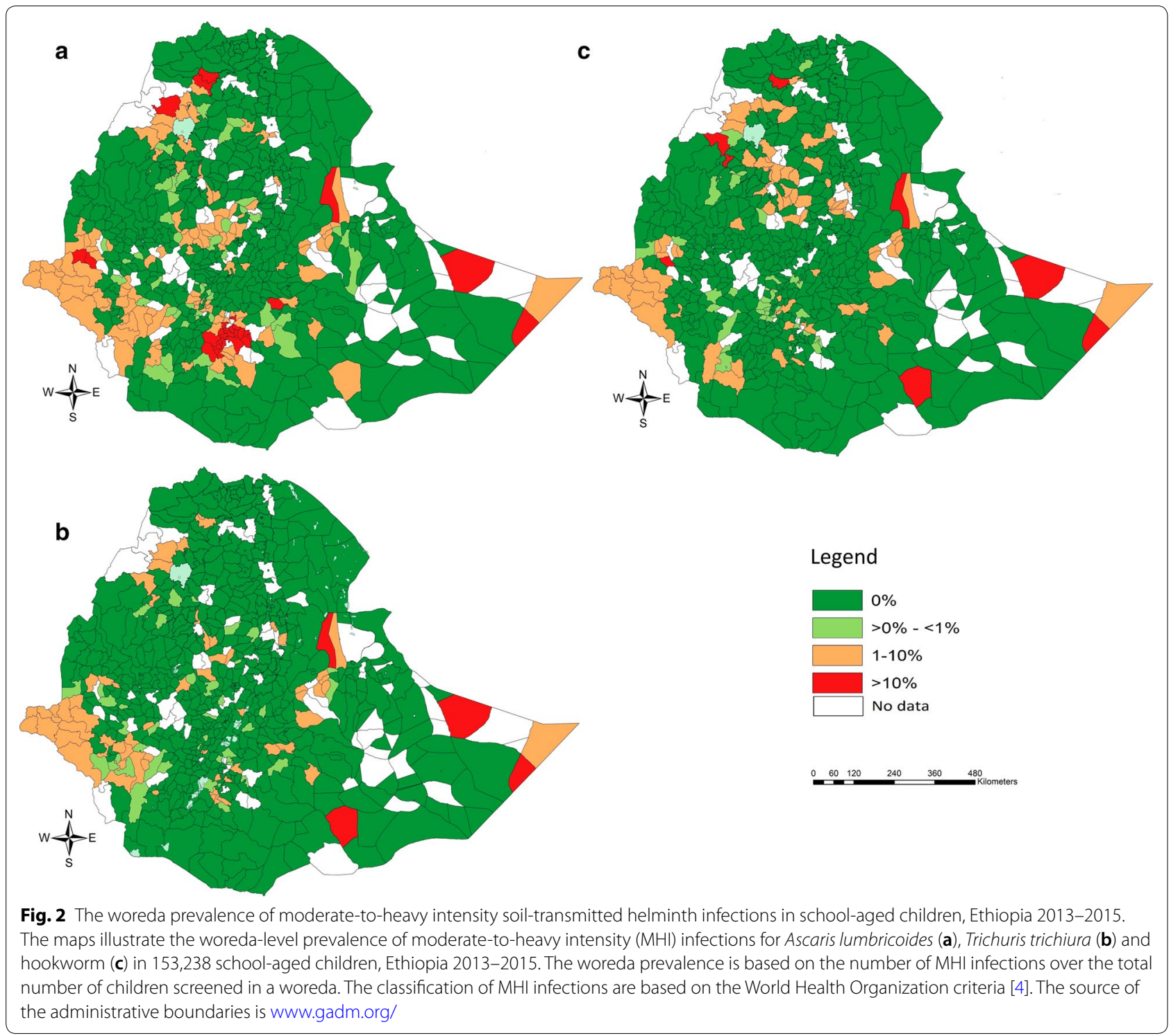

of MDA are covered, and to avoid large-scale deworming being initiated in areas were disease is rare or absent. Although studies highlight that Ethiopia is endemic for both STHs and SCH [5-7], up-to-date maps are currently missing. This study reports the results of the nationwide survey that was designed to provide accurate estimates of the disease distributions at the woreda level, which in turn will inform the national control program against STHs and $\mathrm{SCH}$.

The results of the present study confirm that both STHs and $\mathrm{SCH}$ are prevalent in Ethiopian SAC. The overall prevalence for any STH in this population was estimated to be $21.7 \%$, and $4.1 \%$ for any $\mathrm{SCH}$ in areas where both $\mathrm{SCH}$ species were assessed. To our knowledge, this will be the first large-scale survey conducted at national level. There have been many small-scale studies in different parts of the country that reported prevalence for STH and SCH $[9,11,12,14,15,26]$. However, none of those studies were conducted at a national scale.

Our regional level prevalence for Amhara region is lower than the prevalence report by Nute et al. [8] across the different zones and woredas of the Amhara regional states (STH: 36.5; SCH: 6.9\%) [8]. This difference might be explained by differences in diagnostic methods used (Kato-Katz thick smear $v s$ formol-ether concentration), study population (SAC vs the entire community). Our $18.5 \%$ prevalence for S. haematobium in Somali regional state was in agreement with a previous report by Negussu et al. [13], but it remains unclear why this region is hyperendemic for $\mathrm{SCH}$. However, our sampling strategy prioritized schools close to water bodies, and this region can have highly focal populations which 
Table 2 Variation in prevalence of schistosome infections across Regions/City Administrations, sex and age

\begin{tabular}{|c|c|c|c|c|c|c|c|c|c|}
\hline & \multicolumn{3}{|c|}{ Schistosoma mansoni (\%) } & \multicolumn{3}{|c|}{ Schistosoma haematobium (\%) } & \multicolumn{3}{|c|}{ Any SCH (\%) } \\
\hline & $n$ & Any intensity & $\mathrm{MHI}$ & $n$ & Any intensity & $\mathrm{MHI}$ & $n$ & Any infection & $\mathrm{MHI}$ \\
\hline \multicolumn{10}{|c|}{ Region/City Administration } \\
\hline Addis Ababa & 2718 & 0.1 & 0.1 & 1990 & 0.0 & 0.0 & 1990 & 0.1 & 0.1 \\
\hline Afar & 2377 & 0.3 & 0.1 & 1740 & 0.6 & 0.0 & 1740 & 0.9 & 0.1 \\
\hline Amhara & 25,246 & 3.0 & 1.1 & 18,488 & 0.2 & 0.1 & 18,488 & 3.8 & 1.5 \\
\hline Benishangul-Gumuz & 3109 & $14.9^{*}$ & 5.2 & 2277 & 5.4 & 0.0 & 2277 & 16.9 & 4.0 \\
\hline Dire Dawa & 1701 & 6.2 & 2.6 & 1245 & 0.0 & 0.0 & 1245 & 7.4 & 3.3 \\
\hline Gambela & 2977 & $9.2^{* *}$ & 2.1 & 2180 & 3.8 & 1.3 & 2180 & 12.9 & 3.5 \\
\hline Harari & 1804 & 6.0 & 2.9 & 1321 & 0.0 & 0.0 & 1321 & 6.8 & 3.3 \\
\hline Oromia & 62,520 & 2.1 & 0.9 & 45,785 & 0.0 & 0.0 & 45,785 & 2.0 & 0.9 \\
\hline SNNPR & 37,544 & 2.6 & 1.2 & 27,495 & 0.0 & 0.0 & 27,495 & 3.0 & 1.4 \\
\hline Somali & 966 & 5.9 & 1.3 & 707 & 18.5 & 11.9 & 707 & 21.8 & 13.2 \\
\hline Tigray & 12,276 & $10.8^{*}$ & 3.8 & 8990 & 0.0 & 0.0 & 8990 & 11.0 & 3.9 \\
\hline \multicolumn{10}{|l|}{ Sex } \\
\hline Sex 0 (male) & 76,385 & 3.4 & 1.3 & 56,061 & 0.3 & 0.1 & 56,061 & 3.9 & 1.5 \\
\hline Sex 1 (female) & 76,853 & 3.6 & 1.4 & 56,156 & 0.3 & 0.1 & 56,156 & 4.1 & 1.6 \\
\hline \multicolumn{10}{|l|}{ Age (in years) } \\
\hline $5-9$ & 1201 & 4.1 & 1.4 & 932 & 0.8 & 0.0 & 932 & 3.0 & 0.9 \\
\hline 10 & 23,962 & 3.1 & 1.2 & 17,782 & 0.2 & 0.0 & 17,782 & 3.4 & 1.3 \\
\hline 11 & 38,332 & 2.9 & 1.2 & 28,201 & 0.3 & 0.1 & 28,201 & 3.3 & 1.4 \\
\hline 12 & 45,447 & 3.8 & 1.5 & 32,977 & 0.3 & 0.1 & 32,977 & 4.3 & 1.7 \\
\hline 13 & 27,693 & 3.7 & 1.4 & 20,203 & 0.4 & 0.1 & 20,203 & 4.4 & 1.6 \\
\hline 14 & 15,342 & 4.0 & 1.5 & 11,171 & 0.6 & 0.3 & 11,171 & 4.7 & 1.8 \\
\hline 15 & 1261 & 5.2 & 2.1 & 952 & 2.3 & 1.8 & 952 & 7.7 & 4.1 \\
\hline Total & 153,238 & 3.5 & 1.4 & 112,218 & 0.3 & 0.1 & 112,218 & 4.0 & 1.6 \\
\hline
\end{tabular}

Notes: MHI: moderate-to-heavy intensity; comparisons to the baseline value (Region/City Administration: Afar; sex: sex 0 ; age $=12$ years) that reveal an odds ratio significantly different of one are indicated by at least one asterisk

${ }^{*} P<0.05,{ }^{* *} P<0.01,{ }^{* * *} P<0.001$

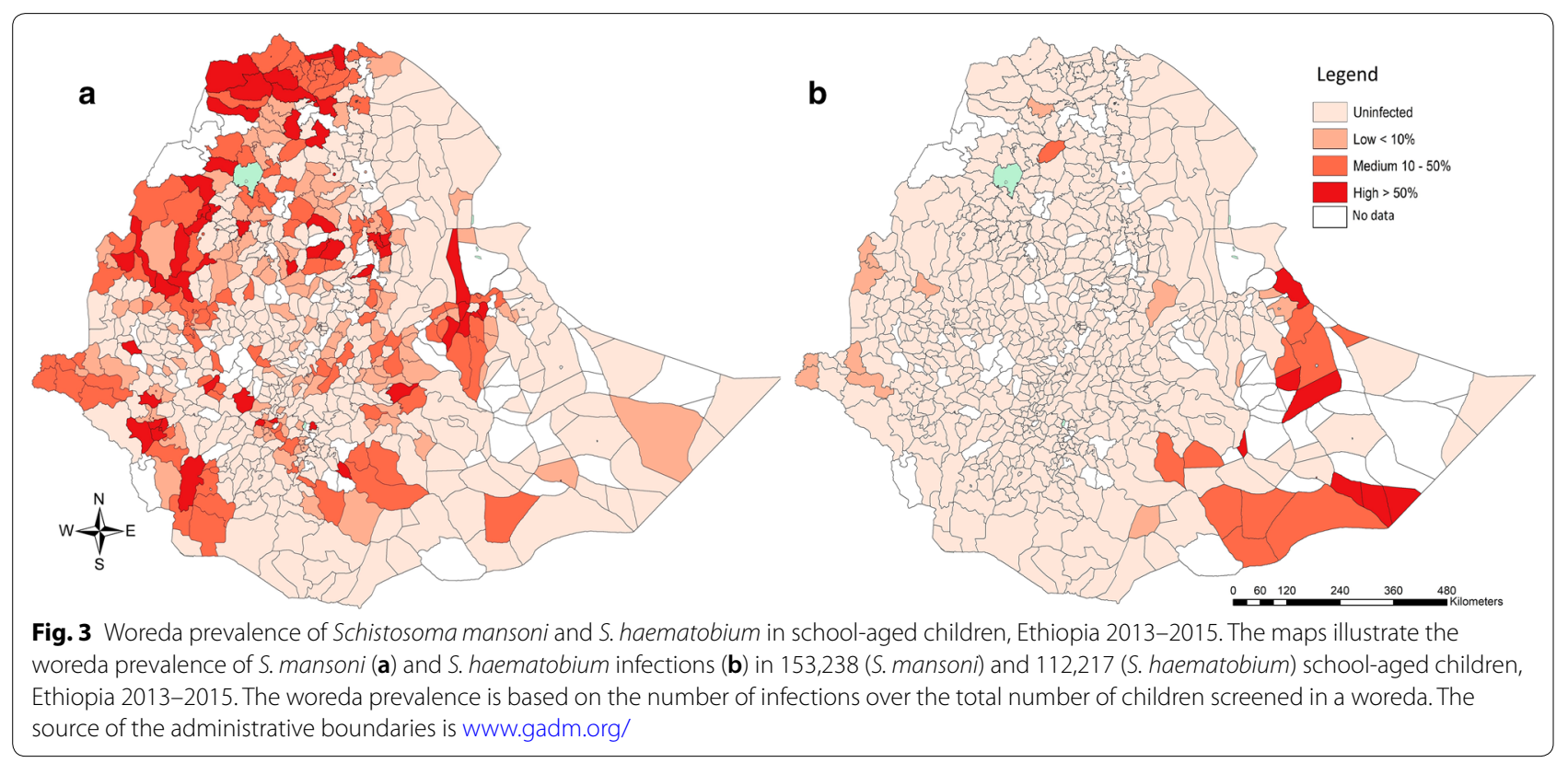



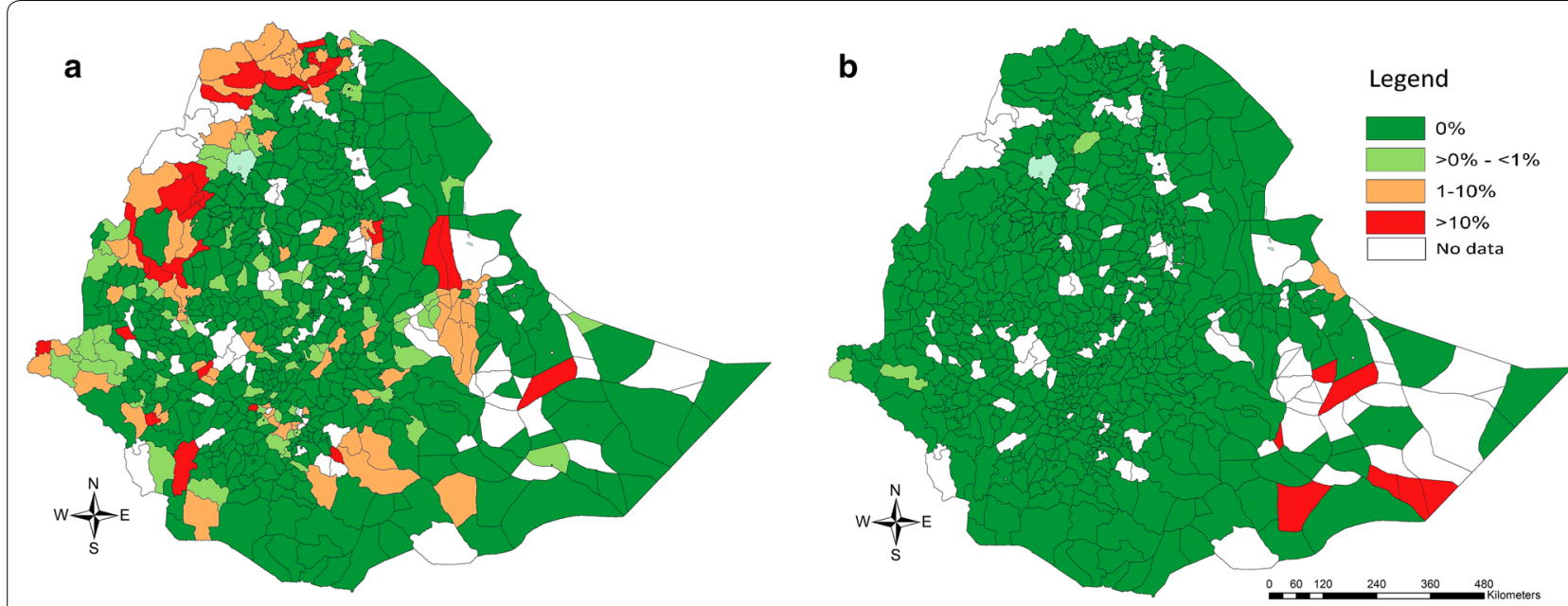

Fig. 4 The woreda prevalence of moderate-to-heavy intensity schistsome infections in school-aged children, Ethiopia 2013-2015. The maps illustrate the woreada prevalence of moderate-to-heavy intensity S. mansoni (a) and S. haematobium infections (b) in 153,238 (S. mansoni) and 112,217 (S. haematobium) school-aged children, Ethiopia 2013-2015. The woreda prevalence is based on the number of MHI infections over the total number of children screened in a woreda. The classification of MHI infections are based on the World Health Organization criteria [4]. The source of the administrative boundaries is www.gadm.org/

Table 3 The mixed soil-transmitted helminth and schistosome infections

\begin{tabular}{ll}
\hline Mixed STH and SCH infections & $n(\%)$ \\
\hline Five helminth species & $1(<0.1)$ \\
All STH and SCH species & $1(0.02)$ \\
Four helminth species & $48(0.8)$ \\
A. lumbricoides + T. trichiura + hookworms + S. mansoni & $45(0.8)$ \\
A. lumbricoides + hookworms + S. mansoni + S. haematobium & $2(<0.1)$ \\
A. lumbricoides + T. trichiura + hookworms + S. haematobium & $1(<0.1)$ \\
Three helminth species & $681(11.8)$ \\
A. lumbricoides + T. trichiura + hookworm & $500(8.7)$ \\
A. lumbricoides + T. trichiura + S. mansoni & $87(1.5)$ \\
A. lumbricoides + hookworms + S. mansoni & $49(0.8)$ \\
T. trichiura + hookworm + S. mansoni & $34(0.6)$ \\
A. lumbricoides + hookworms + S. haematobium & $4(0.1)$ \\
A. lumbricoides + S. mansoni + S. haematobium & $3(<0.1)$ \\
A. lumbricoides + T. trichiura + S. haematobium & $2(<0.1)$ \\
Hookworm + S. mansoni + S. haematobium & $2(<0.1)$ \\
Two helminth species & $5046(87.4)$ \\
A. lumbricoides + T. trichiura & $2232(38.6)$ \\
A. lumbricoides + hookworm & $1371(23.7)$ \\
T. trichiura + hookworm & $462(8.0)$ \\
Hookworm + S. mansoni & $380(6.6)$ \\
T. trichiura + S. mansoni & $287(5.0)$ \\
A. lumbricoides + S. mansoni & $228(3.9)$ \\
A. lumbricoides + S. haematobium & $50(0.9)$ \\
Hookworm + S. haematobium & $19(0.3)$ \\
S. mansoni + S. haematobium & $15(0.3)$ \\
T. trichiura + S. haematobium & $2(<0.1)$ \\
\hline
\end{tabular}

Table 4 The classification of woredas into low, moderate and high endemic for soil-transmitted helminths and schistosome infections

\begin{tabular}{lll}
\hline Level of endemicity & $\begin{array}{l}\text { Soil-transmitted } \\
\text { helminths (\%) }\end{array}$ & Schistosomes (\%) \\
\hline Absence of infections & $37(5.9)$ & $360(57.6)$ \\
Low & $337(53.9)$ & $190(30.4)$ \\
Moderate & $178(28.5)$ & $67(10.7)$ \\
High & $73(11.7)$ & $8(1.3)$ \\
Total & $625(100 \%)$ & $625(100 \%)$ \\
\hline
\end{tabular}

are heavily concentrated around the relatively few rivers. This can provide pockets of high infection in areas that are conducive to snail vector breeding of bulinine snails. The relatively widespread distribution of $S$. mansoni and correlating limited distribution of $S$. haematobium infections in the country is striking. While this paper does not look to identify the underlying reasons, other researchers have identified the impact of altitude and climate on the distribution of the intermediate snail host species (Biomphalaria for S. mansoni and Bulinus for S. haematobium). For example, Kloos et al. [6] identified that most $S$. mansoni infections are found between $1300 \mathrm{~m}$ and 2000 $\mathrm{m}$ with areas outside that range unsuitable for Biomphalaria pfeifferi due to water temperature. Correspondingly, S. haematobium endemic areas are confined to low-lying areas below $800 \mathrm{~m}$ altitude, typically areas only found in Somali and Afar regions. These are in line with the results of the present study. 
Our disease maps highlight that there are large geographical variations in prevalence of infections of any and moderate-to-heavy intensity (Figs. 1-4). This variation can be explained by a variety of factors, including but not limited to differences in climate, population density, and WASH facilities and usage. Alongside this study, associations between WASH and STHs/SCH infections were assessed and reported by Grimes et al. [21]. They concluded that better sanitation was associated with significantly lower A. lumbricoides infection intensity and borderline significant lower hookworm infection intensity. Better hygiene was associated with significantly lower hookworm intensities. However, no significant differences were observed when comparing sanitation and S. mansoni or T. trichiura infections and comparing hygiene and $A$. lumbricoides or T. trichiura infections. Figure 1 highlights that STH-endemic woredas are primarily located in the south, south-west, and north of the country. Dense population and agricultural activities in these areas potentially result in increased exposure to contaminated soil. In the eastern part of the country, infections are less abundant and this may be explained by a dry and arid climate, which does not favour the transmission of these parasites.

The success of the STH and $\mathrm{SCH}$ control programs can be measured by the reduction in moderate-to-heavy intensity infections. Ultimately, the WHO aims to eliminate these diseases as a public health problem, which is defined as a prevalence of moderate-to-heavy intensity infections less than $1 \%$. The prevalence of overall moderate-to-heavy infections above $1 \%$ for both STH and $\mathrm{SCH}$ confirms the importance of the diseases as public health problems in Ethiopia [27]. Yet, it was striking that, despite the lack of large-scale MDA programmes and the abundance of STH and SCH infections, the prevalence of moderate-to-heavy intensity infections was already low (STH: 2\%; SCH: 1.6\%).

The study highlights that $59.3 \%$ and $26.7 \%$ of the woredas are endemic for STH (prevalence $\geq 20 \%$ ) and SCH (prevalence $\geq 10 \%$ ) at a level requiring MDA, meaning that 18 and 14 million SAC are in need of regular deworming through MDA campaigns for $\mathrm{STH}$ and $\mathrm{SCH}$, respectively. The presence of STH and SCH co-infections shows the efficiencies that might be gained with an integrated control strategy. Indeed, in the past, integrated control has led to impressive reductions in prevalence and related morbidity [28]. In addition to integrating control against various parasites, the delivery of different types of parasite control might also be integrated. There are now recommendations to combine MDA with other interventions such as WASH to break parasite transmission and reach elimination [29].

The large areas of overlap in the distributions of the different STH species in Ethiopia could pose a challenge to future control programmes. Currently, the country is using mebendazole in all areas. However, previous studies indicated a differential efficacy between albendazole and mebendazole across STHs. Both drugs are equally efficacious against Ascaris, but albendazole is more efficacious against hookworms whereas mebendazole is more efficacious against Trichuris infections [30].

Following this national survey, Ethiopia has now distributed drugs for 4 years (2015-2019). Overall, the coverage has been high $(>75 \%)$ for both sets of diseases in all woredas [31]. In addition, 175 sentinel schools that represent the different levels of disease endemicity are currently periodically evaluated for the prevalence and intensity of both STH and SCH infections. These sentinel schools are also subjected to a variety of operational research activities in collaboration with national and international partners, with the aim of further improving the control program.

Ethiopia mobilized sufficient financial resources through a range of partners to support its nationwide survey to map the baseline distribution of both diseases. However, the cost of the survey was substantial, and there is a clear need to identify ways to reduce the cost of NTDs mapping, and to reduce the dependency on external partners. In response to this, we verified whether the examination of pooled samples rather than individual samples could be a potential cost-saving strategy in large-scale epidemiological surveys along this nationwide survey [32]. The outcome of this study highlighted that a pooled examination strategy reduced the laboratory time by $70 \%$, but only resulted in an $11 \%$ cost reduction. Moreover, we also recommended pooling for the rapid assessment of infection intensity. However, further investigation is required to determine field procedures and statistical methods for optimal implementation of sample pooling for prevalence assessment.

This study has some limitations. First, the use of single instead of duplicate Kato-Katz thick smear has a clear impact on the prevalence results, as single KatoKatz thick smear is known to be less sensitive than double Kato-Katz thick smear [33]. We opted for a single Kato-Katz for both practical and financial reasons. In general, we chose more children and fewer slides as better than fewer children and more slides. The impact of a single slide on estimates of infection intensity (mean FEC and proportion of moderate-to-heavy infections) is probably less pronounced, since mainly the lowest levels of egg excretion are missed [34]. Secondly, the school selection was biased towards $\mathrm{SCH}$. This strategy was opted, because in contrast to STHs, $\mathrm{SCH}$ has a more focal distribution. Although this will have an impact on our estimates of SCH prevalence, we do not expect that this has a major impact on those for 
the prevalence of STH. Another aspect that may have an impact on the prevalence and hence the decisions made, is the sample size (5 schools per woreda and 50 children). Although this is the sample size recommended by the WHO, it has been shown that this strategy might not be optimal, resulting in unnecessarily administrating drugs or withholding administration of drugs to children in need of treatment, particularly for focally distributed diseases such as SCH [35]. Thirdly, the issues of security, fewer schools overall, and fewer children per school affected the sample size in some remoter areas, particularly in the Somali Region. This may have impacted the accuracy of the estimates in this area. For future surveys we recommend consideration of innovative methods to sample hard-to-reach, mobile and nomadic populations.

The objective of the mapping was to provide a robust estimate of the distribution of SCH and STH in Ethiopia, and hence the number of people that require treatment, with praziquantel (for $\mathrm{SCH}$ ) and albendazole/ mebendazole (for STH). It confirms Ethiopia as having a significant burden of both $\mathrm{SCH}$ and STH. In line with levels of infection found in neighbouring countries, such as Uganda [36], Tanzania [37] and Kenya [38]. Reducing $\mathrm{SCH}$ and $\mathrm{STH}$ infection in populous, high burden countries such as Ethiopia will be crucial for the global community to reach its ambitious 2020 and 2030 goals, under the umbrella of the WHO [1].

\section{Conclusions}

From the regional and global prospective, our study provides optimal baseline information for Ethiopia to implement national $\mathrm{SCH}$ and $\mathrm{STH}$ programs, to enable it to reach the WHO's 2020 goal, reaching $75 \%$ of SAC and $100 \%$ geographical coverage. The nationwide mapping confirms that Ethiopia is endemic to $\mathrm{STH}$ and $\mathrm{SCH}$ infections. The finding from this mapping warrants MDA to SACs living in areas that qualify for treatment. Further mapping initiatives that would provide useful programmatic information, would be the inclusion of preschool age children, adolescent and adult populations to scale up the control program, as well as fine-tuned mapping of hot spots for $\mathrm{SCH}$.

\section{Supplementary information}

Supplementary information accompanies this paper at https://doi. org/10.1186/s13071-020-04317-6.

Additional file 1: Figure S1. Flow diagram of the survey organogram. Survey management and supervision was cascaded in such a way that the Ethiopian Public Health Institute team led and oversaw the whole survey. Regional States supervisors supported their respective teams. The overall mapping implementation was checked by external supervisors.

\section{Abbreviations}

EPG: eggs per gram stool; EPHI: Ethiopian Public Health Institute; FEC: fecal egg counts; FMoE: Federal Ministry of Education; FMoH: Federal Ministry of Health; MDA: mass drug administration; MHI: moderate-to-heavy intensity; NTDs: neglected tropical diseases; SAC: school-aged children; SCH: schistosomes; STH: soil-transmitted helminth; WHO: World Health Organization.

\section{Acknowledgements}

The authors would like to acknowledge the many tens of thousands of school-aged children who permitted to provide samples to allow this survey to happen. We also acknowledge the many parents, schoolteachers, village leaders, health officials, woreda and regional representatives who contributed so much to the success of these surveys.

\section{Authors' contributions}

GT, MF, IG, AF, WH and LD conceived the idea of the study. GT, BL, MF, OS, JG, $J V$ and $A W$ designed the study. SM, HS, AG, YW and KM carried out the field work. GT prepared the manuscript and all authors contributed significantly to this article. All authors read and approved the final manuscript.

\section{Funding}

Financial support for this research was provided by Schistosomiasis Control Initiative and the Partnership for Child Development, both based at Imperial College London and from the Children's Investment Fund Foundation, The End Neglected Diseases Fund and UKAID-DFID. The funders had no role in the study design, data collection and analysis, decision to publish, or preparation of the manuscript.

\section{Availability of data and materials}

The data are owned by the Ethiopian Public Health Institute (EPHI) and are available from EPHI for those researchers who wish to analyse it. Please contact MT (melketadesse@yahoo.com) in the first instance.

\section{Ethics approval and consent to participate}

The study protocol was approved by the Ethiopian Public Health Institute Scientific and Ethical Review Office (reference number SERO-128-4-2005) and the Institutional Review Board of Imperial College London (reference number ICREC_8_2_2). Official letters were written by the Ethiopian Public Health Institute (EPHI) to the Regional Health and Education Bureaus to obtain regional consent. The school head provided written consent on behalf of the students' families or guardians as the survey falls under the mandate of the Ministry of Health. Students provided verbal assent to be included in the survey. All students included in the survey were provided with a single oral dose of 500 mg mebendazole (Janssen Pharmaceutical NV, Beerse, Belgium). In addition, students who tested positive for schistosomiasis were provided with $40 \mathrm{mg}$ $\mathrm{kg} /$ body weight (measured by a dose-pole) of praziquantel (MerckKGaA, Darmstadt, Germany) [1, 4].

\section{Consent for publication}

Not applicable.

\section{Competing interests}

The authors declare that they have no competing interests.

\begin{abstract}
Author details
1 Ethiopian Public Health Institute, PO Box 1242/5654, Addis Ababa, Ethiopia. 2 Department of Virology, Parasitology and Immunology, Faculty of Veterinary Medicine, Ghent University, Merelbeke, Belgium. ${ }^{3}$ Federal Ministry of Health, PO Box 1234, Addis Ababa, Ethiopia. ${ }^{4}$ Department of Civil and Environmental Engineering, South Kensington Campus, Imperial College London, London SW7 2AZ, UK. ${ }^{5}$ Partnership for Child Development, Department of Infectious Disease Epidemiology, St Mary's Campus, Imperial College London, London W2 1PG, UK. ${ }^{6}$ Schistosomiasis Control Initiative, Department of Infectious Disease Epidemiology, St Mary's Campus, Imperial College London, London W2 1PG, UK. ${ }^{7}$ RTI International, Washington D.C, USA.
\end{abstract}

Received: 10 January 2020 Accepted: 25 August 2020

Published online: 01 September 2020 


\section{References}

1. WHO. Accelerating work to overcome the global impact of neglected tropical diseases. A roadmap for implementation. Geneva: World Health Organization; 2012. https://www.who.int/neglected_diseases/NTD_ RoadMap 2012 Fullversion.pdf?ua=1. Accessed 1 Jul 2019.

2. WHO. Preventive chemotherapy in human helminthiasis: coordinated use of anthelminthic drugs in control interventions: a manual for health professionals and programme managers. Geneva: World Health Organization; 2006. https://apps.who.int/iris/bitstream/handle/10665/43545 19241547103_eng.pdf?sequence=1. Accessed 1 Jul 2019.

3. WHO. Fifty-Fourth World Health Assembly. Resolution 54.19. Schistosomiasis and soil-transmitted helminth infections. Geneva: World Health Organization; 2001. https://apps.who.int/iris/bitstream/handle/10665 /260183/WHA54-2001-REC1-eng.pdf?sequence=1\&isAllowed=y. Accessed 1 Jul 2019.

4. WHO. Helminth control in school-age children: a guide for managers of control programmes. Geneva: World Health Organization; 2011. https:// apps.who.int/iris/bitstream/handle/10665/44671/9789241548267_eng. pdf?sequence=1. Accessed $1 \mathrm{Jul} 2019$.

5. Polderman AM. The transmission of intestinal schistosomiasis in Begemder province, Ethiopia. PhD Thesis, Leiden University, Leiden, Netherlands; 1975.

6. Kloos H, Tsong Lo C, Birrie H, Ayele T, Tedla S, Tsegay F. Schistosomiasis in Ethiopia. Soc Sci Med. 1988;26:803-27.

7. Leykun J. Schistosomiasis mansoni and geo-helminthiasis in school children in the Dembia plains, northwest Ethiopia. Ethiop J Heal Dev. 1998;12:3.

8. Nute AW, Endeshaw T, Stewart AEP, Sata E, Bayissasse B, Zerihun M, et al. Prevalence of soil-transmitted helminths and Schistosoma mansoni among a population-based sample of school-age children in Amhara region, Ethiopia. Parasit Vectors. 2018;11:431.

9. Legesse L, Erko B, Hailu A. Current status of intestinal schistosomiasis and soil- transmitted helminthiasis among primary school children in Adwa town, northern Ethiopia. Ethiop J Heal Dev. 2010;24:191-7.

10. Deribe K, Meribo K, Gebre T, Hailu A, Ali A, Aseffa A, et al. The burden of neglected tropical diseases in Ethiopia, and opportunities for integrated control and elimination. Parasit Vectors. 2012;5:240.

11. Mohammed J, Weldegebreal F, Teklemariam Z, Mitiku H. Clinico-epidemiology, malacology and community awareness of Schistosoma mansoni in Haradenaba and Dertoramis kebeles in Bedeno district, eastern Ethiopia. SAGE Open Med. 2018;6:2050312118786748.

12. Tekeste Z, Belyhun Y, Gebrehiwot A, Moges B, Workineh M, Ayalew G, et al. Epidemiology of intestinal schistosomiasis and soil transmitted helminthiasis among primary school children in Gorgora, northwest Ethiopia. Asian Pacific J Trop Dis. 2013;3:61-4.

13. Negussu N, Wali M, Mohamed Y, Ejigu M, Debebe F, Aden S, et al. Prevalence and distribution of schistosomiasis in Afder and Gode zone of Somali region. Ethiopia. J Glob Infect Dis. 2013:5:149.

14. Oswald WE, Stewart AEP, Kramer MR, Endeshaw T, Zerihun M, Melak B, et al. Association of community sanitation usage with soil-transmitted helminth infections among school-aged children in Amhara Region Ethiopia. Parasit Vectors. 2017;10:91.

15. Dejenie T, Asmelash T. Schistosomiasis mansoni among school children of different water source users in Tigray, northern Ethiopia. Momona Ethiop J Sci. 2010;2:49-60.

16. Uniting To Combat Negelected Tropical Diseases. London declaration on neglected tropical diseases. 2012. https://unitingtocombatntds.org/wpcontent/uploads/2017/11/london_declaration_on_ntds.pdf. Accessed 01 Jul 2019.

17. $\mathrm{FMOH}$; Ethiopia. National master plan for neglected tropical disease (2013-2015). 2012. https://ntdenvision.org/sites/default/files/docs/natio nal_ntd_master_plan_ethiopia_2013-2015_1.pdf. Accessed 1 Jun 2019.

18. Gabrielli AF, Montresor A, Chitsulo L, Engels D, Savioli L. Preventive chemotherapy in human helminthiasis: theoretical and operational aspects. Trans R Soc Trop Med Hyg. 2011;105:683-93.

19. Worldometers. Ethiopian Population in 2015. 2019. https://www.world ometers.info/world-population/ethiopia-population/. Accessed 1 Jun 2019.

20. Ash A, Orihel RTC, Savioli L, Sin MA, Montresor A, Renganathan E. Training manual on diagnosis of intestinal parasites based on the WHO bench aids for the diagnosis of intestinal parasites. Geneva: World Health Organization; 2004. http://whqlibdoc.who.int/hq/1998/WHO_CTD_SIP_98.2.pdf.
21. Grimes JET, Tadesse G, Mekete K, Wuletaw Y Gebretsadik A, French MD, et al. School water, sanitation, and hygiene, soil-transmitted helminths, and schistosomes: national mapping in Ethiopia. PLoS Negl Trop Dis. 2016;10(3):e0004515.

22. Speich B, Ali SM, Ame SM, Albonico M, Utzinger J, Keiser J. Quality control in the diagnosis of Trichuris trichiura and Ascaris lumbricoides using the Kato-Katz technique: experience from three randomised controlled trials. Parasit Vectors. 2015;8:82.

23. WHO. Investing to overcome the global impact of neglected tropical diseases: third WHO report on neglected diseases. Geneva: World Health Organization; 2015. https://apps.who.int/iris/bitstream/handle/10665 /152781/9789241564861_eng.pdf?sequence=1. Accessed 1 Jul 2019.

24. Uniting to Combat Neglected Tropical Diseases. Country Leadership and Collaboration on Neglected Tropical Diseases. Third progress report of the London Declaration. 2015. https://unitingtocombatntds.org/wp-content/ uploads/2017/11/3rd_progress_report_english.pdf Accessed 1 Jun 2019.

25. WHO. Schistosomiasis and soil-transmitted helminthiases: number of people treated in 2015. Wkly Epidemiol Rec. 2016;91:585-95.

26. Jemaneh $\mathrm{L}$. Soil transmitted helminthic infection and schistosomiasis mansoni in school children from Chilga district, north west Ethiopia. Ethiop J Heal Sci. 2001;11:2

27. Hotez PJ, Bundy DAP, Beegle K, Brooker S, Drake L, de Silva N, et al. Chapter 24. Helminth infections: soil-transmitted helminth infections and schistosomiasis. In: Jamison DT, Breman JG, Measham AR, Alleyne G, Claeson $M$, Evans DB, et al, editors. Disease control priorities in developing countries. Washington (DC): The International Bank for Reconstruction and Development / The World Bank; 2006.

28. Kaatano GM, Siza JE, Mwanga JR, Min DY, Yong TS, Chai JY, et al. Integrated schistosomiasis and soil-transmitted helminthiasis control over five years on Kome Island, Tanzania. Korean J Parasitol. 2015;53:535-43.

29. Lo NC, Addiss DG, Hotez PJ, King CHJ, Stothard JR, Evans DS, et al. A call to strengthen the global strategy against schistosomiasis and soil-transmitted helminthiasis: the time is now. Lancet Infect Dis. 2017:17:e64-9.

30. Vercruysse J, Behnke JM, Albonico M, Ame SM, Angebault C, Bethony $\mathrm{JM}$, et al. Assessment of the anthelmintic efficacy of albendazole in school children in seven countries where soil-transmitted helminths are endemic. PLoS Negl Trop Dis. 2011;5:e948.

31. Negussu N, Mengistu B, Kebede B, Deribe K, Ejigu E, Tadesse G, et al. Ethiopia schistosomiasis and soil-transmitted helminthes control programme: progress and prospects. Ethiop Med J. 2017;55:75-80.

32. Leta GT, French M, Dorny P, Vercruysse J, Levecke B. Comparison of individual and pooled diagnostic examination strategies during the national mapping of soil-transmitted helminths and Schistosoma mansoni in Ethiopia. PLoS Negl Trop Dis. 2018;12:e0006723.

33. Nikolay B, Brooker SJ, Pullan RL. Sensitivity of diagnostic tests for human soil-transmitted helminth infections: a meta-analysis in the absence of a true gold standard. Int J Parasitol. 2014;44:765-74.

34. Bärenbold O, Raso G, Coulibaly JT, N'Goran EK, Utzinger J, Vounatsou P. Estimating sensitivity of the Kato-Katz technique for the diagnosis of Schistosoma mansoni and hookworm in relation to infection intensity. PLoS Negl Trop Dis. 2017;11:e0005953.

35. Tchuem Tchuenté L-A, Stothard JR, Rollinson D, Reinhard-Rupp J. Precision mapping: an innovative tool and way forward to shrink the map, better target interventions, and accelerate toward the elimination of schistosomiasis. PLoS Negl Trop Dis. 2018;12:e0006563.

36. Kabatereine NB, Brooker S, Tukahebwa EM, Kazibwe F, Onapa AW. Epidemiology and geography of Schistosoma mansoni in Uganda: implications for planning control. Trop Med Int Health. 2004;9:372-80.

37. Clements ACA, Nyandindi U, Fenwick A, Brooker S, Blair L. Bayesian spatial analysis of a national urinary schistosomiasis morbidity questionnaire in Tanzania, east Africa. Int J Parasitol. 2008;38:401-15.

38. Smith JL, Sturrock HJW, Assefa L, Nikolay B, Njenga SM, Kihara J, et al. Factors associated with the performance and cost-effectiveness of using lymphatic filariasis transmission assessment surveys for monitoring soil-transmitted helminths: a case study in Kenya. Am J Trop Med Hyg. 2015;92:342-53.

\section{Publisher's Note}

Springer Nature remains neutral with regard to jurisdictional claims in published maps and institutional affiliations. 